\title{
Heavy ion collision experiments at NICA
}

\section{Vladimir Kekelidze*}

Joint Institute for Nuclear Research, 141980 Dubna, Russia

E-mail: kekelidze@jinr.ru

\section{Vadim Kolesnikov}

Joint Institute for Nuclear Research, 141980 Dubna, Russia

E-mail: Vadim.Kolesnikov@cern.ch

\section{Richard Lednicky}

Joint Institute for Nuclear Research, 141980 Dubna, Russia

E-mail: lednicky@fzu.cz

\section{Victor Matveev}

Joint Institute for Nuclear Research, 141980 Dubna, Russia

E-mail: matveev@inr.ac.ru

\section{Alexander Sorin}

Joint Institute for Nuclear Research, 141980 Dubna, Russia

National Research Nuclear University (MEPhI), 115409 Moscow, Russia

E-mail: sorinatheor.jinr.ru

\section{Grigoriy Trubnikov}

Joint Institute for Nuclear Research, 141980 Dubna, Russia

E-mail: trubnikovejinr.ru

The NICA (Nuclotron-based Ion Collider fAcility) project is under realization at the Joint Institute for Nuclear Research (JINR, Dubna). The main goal of the project is an experimental study of hot and dense strongly interacting matter in heavy ion (up to $\mathrm{Au}$ ) collisions at nucleon-nucleon center-of-mass energies up to $11 \mathrm{GeV}$. The physics program will be performed in two experiments, BM@N (Baryonic Matter at Nuclotron) at beams extracted from the Nuclotron, and MPD (MultiPurpose Detector) at the NICA collider. This program covers a variety of phenomena in strongly interacting matter of the highest net baryon density.

XXXIX International Conference on High Energy Physics

4-11 July 2018

Seoul, Korea

\footnotetext{
* Speaker.
} 


\section{Introduction}

Heavy ion collisions is the unique tool of creating and studing in the laboratory nuclear matter under extreme conditions. A new accelerator facility NICA [1], which is under construction at JINR (Dubna), will provide a broad list of research opportunities: study of the nuclear equation-ofstate at the highest net baryon density, in-medium modifications of hadron properties, phase transformations and critical behavior in the strongly-interacting matter, properties of hyperon-nucleon interaction, and the spin structure of the nucleon. In order to archive these goals a variety of beam species will be available at NICA utilizing both the collider and fixed target collision mode. During the first period of the project realization the emphasis will be put on the study of the excitation function of hadroproduction, investigation of the reaction dynamics, event-by-event fluctuations and correlations, production of multistrange hyperons (including the threshold region) and hypernuclei.

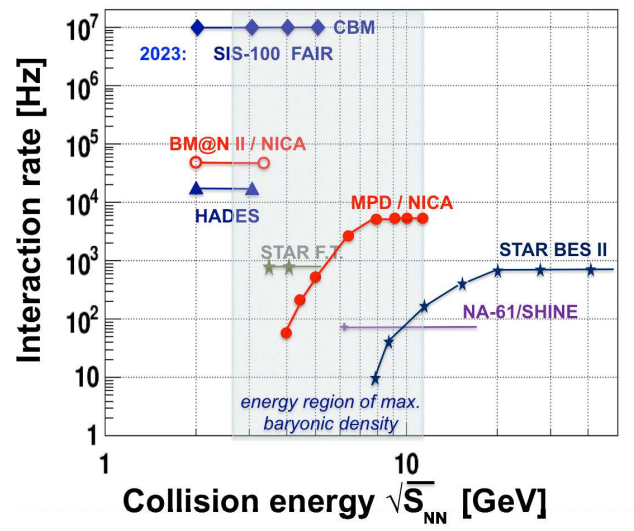

Figure 1: Typical event rates of heavy-ion collisions in the energy range of high net baryon density. The values for the NICA experiments are shown by red circles.

\section{Accelerator complex NICA}

The NICA complex, composed of a chain of accelerators, will provide a variety of ion species from polarized protons and deuterons to heavy ions with a design intensity of $2 \cdot 10^{9}$ particles per cycle for gold ions. Before the collision happens, charged particles travel through the heavy-ion source (or source of polarized particles), a linear accelerator, a booster, the Nuclotron accelerator, and finally, two storage rings of a collider (of about $503 \mathrm{~m}$ circumference each) with two focusing points for heavy-ion and polarized particle collisions. Thanks to the modern electron and stochastic cooling systems at NICA, the achieved collider luminosity for gold ions will be from $6 \cdot 10^{25}$ to $10^{27} \mathrm{~cm}^{-2} \mathrm{~s}^{-1}$ (energy range $4<\sqrt{s_{N N}}<11 \mathrm{GeV}$ ), while that for polarized protons and deuterons will achieve of $10^{32} \mathrm{~cm}^{-2} \mathrm{~s}^{-1}$ (with the center-of-mass energy for $\mathrm{p}+\mathrm{p}$ collisions up to $27 \mathrm{GeV}$ ). The upgraded Nuclotron accelerator extracted beams have the maximum energy per nucleon of 5.8 $\mathrm{GeV}$ for $\mathrm{A} / \mathrm{Z}=0.5$ specie and of $4.5 \mathrm{GeV}$ for ${ }^{+79} \mathrm{Au}$ nuclei. The event rates achieved at NICA are shown in Fig. 1 together with the numbers for SPS, FAIR, and RHIC experiments. As one can 
see, in the region of the highest net baryon density (shaded area) the event rate up to $7 \mathrm{kHz}$ can be achieved at the NICA collider.

Serial fabrication of the magnets for the NICA facility is close to the completion and the process of commissioning of the booster has already started. The construction of the new NICA infrastructure, including buildings, transfer lines, service and supply systems is progressing according to plan. The first heavy-ion collision at the NICA collider is expected in 2021 with a start-up version of the project (i.e. the peak luminosity reduced to the level of $5 \cdot 10^{25} \mathrm{~cm}^{-2} \mathrm{c}^{-1}$ ). The completion of the NICA commissioning with its nominal luminosity value is scheduled for 2023.

\section{Detectors at NICA}
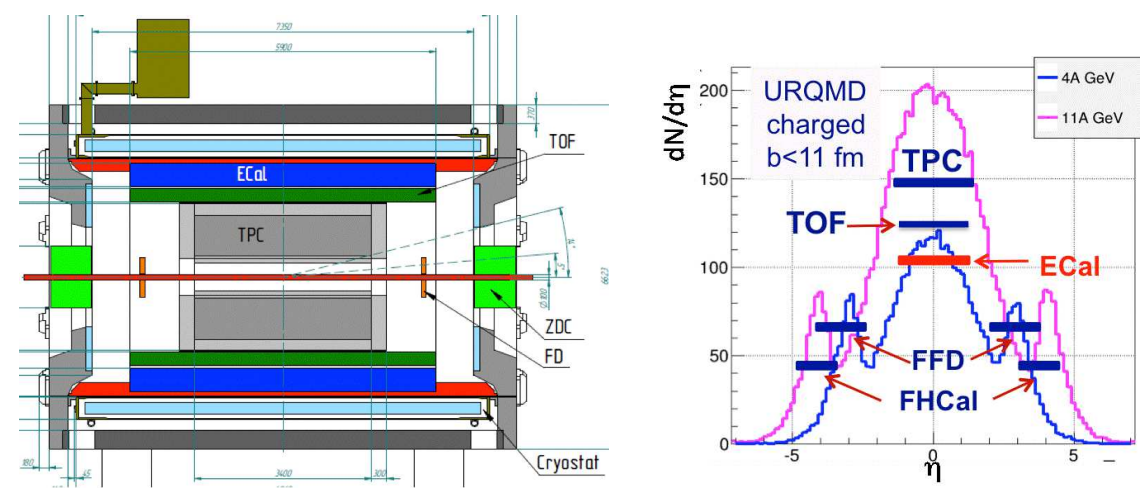

Figure 2: Left: A view of the MPD detector at NICA. Right: MPD setup pseudorapidity coverage.

In order to fulfill all the NICA physics goal the MultiPurpose Detector (MPD) at NICA is designed as a large uniform acceptance spectrometer capable of detecting large variety of hadrons and nuclei, as well as electrons and gammas [2]. It consists of an assembly of various subsystems (see Fig.2, left panel) enclosed in a superconducting solenoid which creates a high homogeneity magnetic field of up to $0.6 \mathrm{~T}$. The main charged particle tracking device in MPD is a cylindrical Time-Projection Chamber (TPC) of $2.8 \mathrm{~m}$ (diameter) by $3.4 \mathrm{~m}$ (length), which can reconstruct a 3D image of the trajectory within the pseudorapidity range $|\eta|<1.6$. With 53 measured space points per a track at midrapidity, TPC will enable particle identification via the specific energy loss $(d E / d x)$ measurement with a precision better than $8 \%$. The TOF system made of multigap resistive plate chambers (MRPC) with the intrinsic time resolution of about 65 ps enables charged hadron discrimination at momenta $p<4 \mathrm{GeV} / \mathrm{c}$. Arrays of quartz counters (FD) of the $40 \mathrm{ps}$ time resolution can provide fast timing and triggering signals. The Electromagnetic Calorimeter (ECAL) made of "shashlyk" lead-scintillator towers comprises about 43000 modules of 18 radiation length thickness forming a barrel shape with projective geometry directing to the MPD center. Two arms of hadron calorimeters (FHCAL), made of 44 modules of lead-scintillator sampling calorimeters and covering the peudorapidity range $2.8<|\eta|<4.5$, will be used for the offline centrality estimate and event plane analysis. Figure 2 (right panel) indicates the whole phase-space coverage of the MPD setup for minimum bias $\mathrm{Au}+\mathrm{Au}$ reactions at the lowest and the highest collision energy. A more detailed description of the detector components can be found elsewhere [3]. After completing 
of R\&D and preparation of Technical Design Reports the team is now in the process of industrial production of detector elements.

BM@N (Baryonic Matter at Nuclotron) is a fixed target experiment at the Nuclotron accelerator [4]. Its experimental setup is drawn in Fig. 3. Charged particles will be measured with a set of 12 Gaseous Electron Multipliers (GEM) chambers located downstream of the target inside an analyzing magnet of $0.8 \mathrm{~T}$ complemented by drift/pad detectors $(\mathrm{DCH}, \mathrm{CPC})$ situated outside the magnet. Time-of-flight detectors (TOF1,2) made of resistive plate chambers with a strip read-out enable discrimination between particle species up to a few $\mathrm{GeV} / \mathrm{c}$ momentum. The recoil detector at the backward hemisphere is designed for event plane determination. Zero degree calorimeter (ZDC) is constructed to measure the energy of forward going particles and calculate the degree of collision centrality. First two technical runs with a part of the BM@N setup have already been carried out, and the program, including data taking with carbon and argon beams, was dedicated to tuning of the beam line, GEM and MRPC chambers, trigger and data acquisition system. The first physics run with $\mathrm{Au}$ ions is planed for 2020 after the NICA booster commissioning.

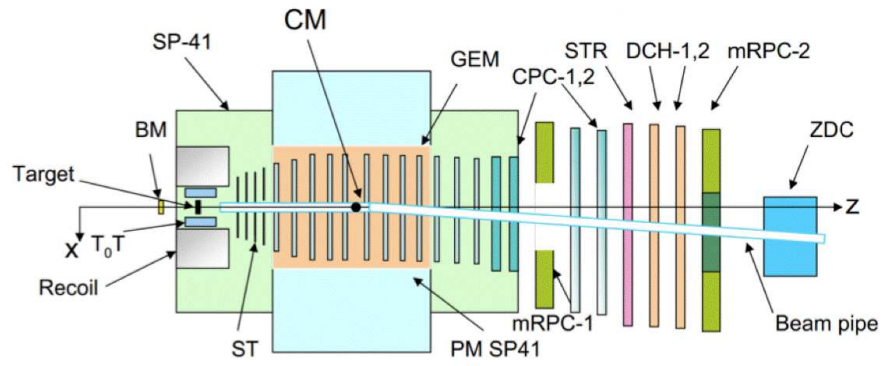

Figure 3: A scheme of the BM@N experiment at the Nuclotron

\section{Conclusion}

Realization of the NICA project at JINR can extend the experimental study of stronglyinteracting matter to the region of the highest net baryon density. The construction of the accelerator complex and experimental setups at NICA is going close to the schedule enabling the start-up configurations of the collider and detectors be ready in 2021.

\section{References}

[1] V. D. Kekelidze, R. Lednicky, V. A. Matveev, I. N. Meshkov, A. S. Sorin and G. V. Trubnikov, Eur. Phys. J. A (2016) 52: 211.

[2] V. Golovatyuk, V. Kekelidze, V. Kolesnikov, O. Rogachevsky, and A. Sorin, Eur. Phys. J. A (2016) 52: 212.

[3] K.U. Abraamyan et al., Nucl. Instrum. Meth. A 628, 99 (2011).

[4] Mikhail Kapishin for the BM@N Collaboration, Eur. Phys. J. A (2016) 52: 213. 